\title{
Low CCL19 Expression Is Associated With Adverse Clinical Outcomes For Patients With Advanced Stage Follicular Lymphoma
}

\section{Yu Zhou}

Cancer Hospital Chinese Academy of Medical Sciences

\section{Shasha Wang}

Cancer Hospital Chinese Academy of Medical Sciences

\section{Yunxia Tao}

Cancer Hospital Chinese Academy of Medical Sciences

\section{Haizhu Chen}

Cancer Hospital Chinese Academy of Medical Sciences

\section{Yan Qin}

Cancer Hospital Chinese Academy of Medical Sciences

Xiaohui He

Cancer Hospital Chinese Academy of Medical Sciences

\section{Shengyu Zhou}

Cancer Hospital Chinese Academy of Medical Sciences

\section{Peng Liu}

Cancer Hospital Chinese Academy of Medical Sciences Jianliang Yang

Cancer Hospital Chinese Academy of Medical Sciences

\section{Sheng Yang}

Cancer Hospital Chinese Academy of Medical Sciences

\section{Lin Gui}

Cancer Hospital Chinese Academy of Medical Sciences

Ning Lou

Cancer Hospital Chinese Academy of Medical Sciences

\section{Zhishang Zhang}

Cancer Hospital Chinese Academy of Medical Sciences

\section{Jiarui Yao}

Cancer Hospital Chinese Academy of Medical Sciences

\section{Xiaohong Han}

Peking Union Medical College Hospital

Yuankai Shi ( $\nabla$ syuankai@cicams.ac.cn ) 


\section{Research}

Keywords: follicular lymphoma, CCL19, prognosis, survival outcome, chemokine

Posted Date: August 6th, 2021

DOI: https://doi.org/10.21203/rs.3.rs-764209/v1

License: (c) (i) This work is licensed under a Creative Commons Attribution 4.0 International License. Read Full License 


\section{Abstract}

Background: This study aimed to explore the interactions and relationships of genes and recognize the hub genes associated with prognosis in follicular lymphoma (FL) treated with first-line rituximab combined with chemotherapy.

Method: RNA sequencing data of dataset GSE65135 $(n=24)$ were included in differentially expressed genes (DEGs) analysis. Weighted gene co-expression network analysis (WGCNA) was applied for exploring the coexpression network and identifying hub genes. Validation of hub genes expression and prognosis were applied in dataset GSE119214 $(n=137)$ and patient cohort of Cancer Hospital, Chinese Academy of Medical Sciences \& Peking Union Medical College $(n=32)$, respectively, by analyzing RNAseq expression data and serum protein concentration quantified by ELISA. CIBERSORT was applied for tumorinfiltrating immune cells (TIICs) subset analysis.

Results: A total of 3260 DEGs were obtained, with 1861 genes upregulated and 1399 genes downregulated. Using WGCNA and Cytoscape analysis, eight hub genes, PLA2G2D, MMP9, PTGDS, CCL19, NFIB, YAP1, RGL 1, and TIMP3 were identified. Kaplan-Meier analysis and multivariate COX regression analysis indicated that $C C L 19$ independently associated with overall survival (OS) for FL patients treated with rituximab and chemotherapy $(\mathrm{HR}=0.47,95 \% \mathrm{Cl}[0.25-0.86], \mathrm{p}=0.014)$. Higher Serum CCL19 concentration was associated with longer progression-free survival ( $P F S, p=0.014)$ and OS $(p=0.039)$. TIICs subset analysis showed that CCL19 expression had a positive correlation with monocytes and macrophages $\mathrm{M} 1$, and a negative correlation with naïve B cells and plasma cells.

Conclusion: CCL 19 expression was associated with survival outcomes and might be a potential prognostic biomarker for FL treated with first-line immunochemotherapy.

\section{Introduction}

Follicular lymphoma $(\mathrm{FL})$ is the second common B-cell lymphoma that compromises about $12 \%$ of all mature non-Hodgkin lymphoma $(\mathrm{NHL})[1,2=. \mathrm{FL}$ is an indolent $\mathrm{NHL}$ and is characterized with repeatedly relapse in the long disease history in most patients[3=. Although the advent of rituximab improved the progression-free survival (PFS) and overall survival (OS) of $F L[4,5=$, still approximately $20 \%$ patients with FL relapsed with two years after first-line chemoimmunotherapy $[6=$, which lead to a dismal prognosis. Therefore, identifying the high-risk patients with suboptimal treatment response before treatment and improve their survival outcomes are necessary for the management of FL.

Most previous studies focused on screening differential expression genes and establishing gene models in $\mathrm{FL}[7,8=$. However, the interconnectivity and co-expression of the genes was ignored. Weighted gene co-expression network analysis (WGCNA) is a useful method to explore the interactions and relationships within genes and can help to recognize the hub genes associated with clinical characteristics $[9,10=$. WGCNA can recognize the core gene and provide information for potential clinical prognosis biomarkers and has been widely used in cancer genome-related researches, including other subtypes of lymphoma 
$[11-13=$. In this study, we sought to identify the hub genes for FL using WGCNA conducted in a cohort containing FL samples and normal samples. We focused on the prognosis value of the identified hub genes. The association of hub genes and survival outcomes was validated in two independent cohorts including both tumor samples and serum samples of $\mathrm{FL}$ patients receiving standard first-line treatment, respectively. We aimed to raise new potential biomarkers for $\mathrm{FL}$, which could bring more insight for diagnosis and clinical treatment in FL.

\section{Materials And Methods}

\section{Patients and datasets}

GSE65135 containing RNA sequencing (RNA-seq) data of 24 cases, including 10 normal tonsil tissue (control group) and $14 \mathrm{FL}$ tissue (cancer group) obtained from Gene Expression Omnibus (GEO) database (https://www.ncbi.nlm.nih.gov/geo/) was used for exploring the differential expressed genes (DEGs). GPL570 (Affymetrix Human Genome U133 Plus 2.0 Array) was used for this dataset and the annotation for the gene IDs was conducted using the hgu133plus2.db package in R software (Vienna, Austria. https://www.R-project.org/). The Affy package in R was applied for raw data quality control, preconditioning and sorting out[14=. The expression of repeated genes was expressed as mean values.

To validate the prognostic value of hub genes identified from GSE65135, GEO database was searched using the term "follicular lymphoma", with organism restricted to "Homo sapiens". Up to January 9th, 2021, a total of $147 \mathrm{FL}$ related GEO series was screened. Datasets of expression data with complete prognostic information were included in the validation cohort. Only one dataset, GSE119214 contained expression data and survival data (FFS and OS) of pre-treated FFPE samples of $137 \mathrm{FL}$ patients treated with rituximab and chemotherapy and was included in the validation cohort. GPL13938 (Illumina HumanHT-12 WG-DASL V4.0 expression beadchip) was selected for this microarray dataset and gene IDs were mapped to the microarray probes using the annotation information provided on GEO dataset.

Additionally, pre-treated serum samples of patients with FL treated with first-line chemoimmunotherapy in the Cancer Hospital, Chinese Academy of Medical Sciences \& Peking Union Medical College (CAMS \& PUMC) from 2014 to 2018 were used for further validation of hub genes with clinical significance identified from GSE119214. Inclusion criteria including: patients with newly diagnosed FL, receiving standard R-CHOP or R-CHOP-like chemoimmunotherapy regimen, with follow-up information. All the samples were obtained before the initiation of treatment and were with informed consent. Sample collection was approved by the Hospital's Protection of Human Subjects Committee. All data collected were anonymized. This study was approved by the medical ethics committee of Cancer Hospital, CAMS \& PUMC (No. 19/088-1873). The cutoff date for follow-up was July 2nd, 2021. A total of 32 samples were included in this cohort (CHCAMS cohort). The patient characteristics of GSE119214 and CHCAMS cohort were shown in supplementary Table S1.

\section{Differentially expressed genes screening}


The limma package in $\mathrm{R}$ was used for screening the differentially expressed genes (DEGs) between control group and cancer group in GSE65135 [15=. The thresholds for DEGs were as follows: (1) $p<0.05$; (2) $\log 2$ (fold change) $>1$ or <-1. Volcano plot and heatmap generated by the ggplot2 package were displayed to show the DEGs.

\section{Gene enrichment analysis}

Gene Ontology (GO) analysis was performed for analyzing the unique biological significance based on DEGs. The Kyoto Encyclopedia of Genes and Genomes (KEGG) analysis were performed to find the important pathways among the DEGs. The clusterProfiler package in R was used for analyzing GO annotation and KEGG pathway[16=. Gene Set Enrichment Analysis (GSEA) software (http://www.gsea$\mathrm{msigdb.org/gsea/)} \mathrm{was} \mathrm{applied} \mathrm{to} \mathrm{analyze} \mathrm{the} \mathrm{signal} \mathrm{pathway} \mathrm{enriched} \mathrm{in} \mathrm{tumor} \mathrm{samples[17=.} \mathrm{The}$ GOplot package in $\mathrm{R}$ was used for result visualization[18=.

\section{Coexpression Network Construction and hub gene selection}

WGCNA package in $\mathrm{R}$ was applied for exploring the coexpression network and identifying hub genes[9, $10=$. Expression data of DEGs were input into R and undergoing quality check before coexpression analysis. Samples and genes with poor quality would be excluded. Expression data of qualified samples were included in the analysis and similar matrix was constructed by calculating the Pearson correlation coefficient of two genes. Soft thresholding power was explored to ensure a scale-free network. The mean connectivity and scale independence of network modules were analyzed using gradient under soft thresholding power ranging from 1 to 20 . Hierarchical clustering dendrogram summarized the gene modules. The minimum number of each gene module was set at 50 to ensure the reliability of each module. Heatmap and topological overlap matrix (TOM) plot were drawn to display the intensity of interaction among the modules.

The genes of modules were then used to construct the functional protein network. To further analyze the hub genes of the network, edges and nodes of network were output under certain threshold value (0.4) to Cytoscape software (version 3.8.0; http://www.cytoscape.org/) for analyzing and visualizing[19=. CytoHubba plugin in Cytoscape was applied for analyzing the degree of connectivity for each gene. The degree of connectivity set to rank genes. Genes with connectivity degree over 20 were identified and were considered to be hub genes. Identified hub genes were uploaded in the Search Tool for the Retrieval of Interacting Genes/Proteins (STRING) online database (version 11.0, https://string-db.org/) for analyzing the protein-protein interaction (PPI).

\section{Validation of hub genes}

To validate the prognostic power of hub genes, Kaplan-Meier analysis were applied using the data of GSE119214 and CHCAMS cohort. The best expression cut-off value for each hub gene for OS was obtained by the maxstat package of R. To identify the independent prognostic value of hub genes, multivariate analysis was applied. 
To further explore mRNA levels of hub genes, ONCOMINE database (http://www.oncomine.org), a publicly accessible online cancer microarray database that facilitates the discovery of genome wide expression analyses, was applied. Student's t test was conducted for comparing the mRNA level of cancer specimens and normal control datasets. Fold change value was set as 2 and $p$ value $<0.05$ were considered as significant.

\section{Measurement of serum CCL19 concentration}

Peripheral blood samples were obtained before first-line treatment, and serum was extracted and stored at $-80^{\circ} \mathrm{C}$ until use. Serum CCL19 concentrations were quantified using the CCL19 human enzyme-linked immunosorbent assay (ELISA) kit according to the manufacturer's instructions (Mlbio, Enzyme-linked Biotechnology Co., Shanghai, China).

\section{CIBERSORT analysis}

CIBERSORT (https://cibersort.stanford.edu/) was applied to analyze the association of tumor microenvironment and the expression of CCL19 $[20=$. The proportion of tumor-infiltrating immune cells (TIICs) of each samples in GSE119214, including naive B cells, memory B cells, plasma cells, CD8 + T cells, naive CD $4+T$ cells, CD 4 + resting memory T cells, CD 4 + memory-activated T cells, follicular helper $T$ cells, Treg cells, $\gamma \delta T$ cells, resting natural killer cells, activated natural killer cells, monocytes, M0 macrophages, M1 macrophages, M2 macrophages, resting dendritic cells, activated dendritic cells, resting mast cells, activated mast cells, eosinophils, and neutrophils, was calculated.

\section{Statistical analysis}

Univariable analysis was performed using Kaplan-Meier survival analysis with log-rank test. Multivariable analysis was performed using COX regression model. Correlations between two groups were calculated with Spearman's coefficient $(R)$. Comparison of two groups was performed using Mann-Whitney-Wilcoxon test and comparison of multiple groups was performed using Kruskal-Wallis test. All statistical analyses were performed and visualized by R studio software (version 4.0.3, https://www.r-project.org/) and GraphPad PRISM (version 8.0.2). Two-side $p$ value $<0.05$ was considered as statistically significant.

\section{Result}

\section{Overview of Differentially Expressed Genes}

In differential expression analysis, a total of 3260 differentially expressed genes (DEGs) were obtained, of which 1861 genes were upregulated and 1399 genes were downregulated in tumor samples compared with normal samples (Fig. 1A to B).

To further analyze the biological function of the DEGs, the upregulated and downregulated genes were further analyzed with GO and KEGG enrichment analysis, respectively. Functional enrichment analysis was performed in the biological process. Up-regulated DEGs were enriched in extracellular matrix organization, extracellular structure organization and regulation of vasculature development, while down- 
regulated DEGs were enriched in nucleosome assembly, chromatin assembly or disassembly and chromatin assembly (Fig. 1C and 1D). KEGG analysis showed that the upregulated DEGs were enriched in PI3K-AKT signaling pathway, cytokine-cytokine receptor interaction and cell adhesion molecules. The down-regulated DEGs were involved in alcoholism, neutrophil extracellular trap formation and systemic lupus erythematosus (Fig. 1E and 1F).

As all the genes included in the GO and KEGG analysis were selected under the threshold set artificially ( $p$ $<0.05$; $\log 2$ [fold change] $>1$ or $<-1$ ), the results might be different under different thresholds. Therefore, all expression dataset was included in GSEA analysis. Results showed that IL-6/JAK/STAT3 signaling, complement and mitotic spindle were three pathways that mostly enriched in tumor samples (supplementary Figure S1).

\section{Weighted Gene Correlation Network Analysis}

To explore the key modules and hub genes in FL, WGCNA was performed for network construction to find highly-correlated genes. All the cancer samples and controlled samples were included in the analysis after quality check (supplementary Figure S2). Soft thresholding power was set at 6 to ensure a scale-free network, with scale-free $R^{2}=0.88$ and mean $k=134$ (supplementary Figure S3). Gene modules were explored and the identified modules were showed in the hierarchical cluster tree (Fig. 2A). A total of 3260 DEGs were allocated in three modules, with 633 genes in blue gene module, 209 genes in brown gene module and 2418 genes in gene module. Heatmap plot show the topological overlap matrix (TOM) among all genes and the interactive relationships between all three coexpression modules (Fig. 2B). Figure $2 \mathrm{C}$ illustrated the relationship of the modules with FL. According to the Pierson correlation coefficient between a module and sample feature for each module, turquoise module was closely associated with $\mathrm{FL}(R=0.99)$ and the genes of this module were further analyzed. The functional enrichment of genes in turquoise module was further explored. GO analysis showed that cell chemotaxis, extracellular matrix organization and extracellular structure organization were the most enriched pathways of genes in turquoise module. KEGG analysis indicated that genes of turquoise module were mostly enriched in alcoholism, neutrophil extracellular trap formation and systemic lupus erythematosus pathways (supplementary Figure S4).

\section{Identification of hub genes}

The threshold of edge weight was set to be more than 0.4 to localized hub genes. A total of 1791 edges and 382 nodes of turquoise module were included in Cytoscape analysis. The genes involved in the network were ranked by degree calculated in cytoHubba plugin to explore potential hub genes. Supplementary Figure S5A showed the top 50 genes ranked by degree in turquoise module. The top 8 genes with a threshold degree $>20$ were identified and considered as hub genes ( $P L A 2 G 2 D, M M P$, PTGDS, CCL 19, NFIB, YAP1, RGL 1, and TIMP3). The PPI network of hub genes was illustrated in supplementary Figure S5B.

\section{RNA expression analysis for the validation of hub genes}


Oncomine database was applied to analyze the mRNA expression of the eight hub genes. Supplementary Figure $\mathrm{S} 6$ illustrated the expression of the hub genes among different cancer types. All of the eight hub genes were overexpressed and MMP9, NFIB, YAP1, RGL 1 and TIMP3 expression were down-regulated across different datasets.

Next, the expression and prognostic value of hub genes were validated in another independent GEO dataset with expression profiling and survival information of $137 \mathrm{FL}$ samples (GSE119214) [21=. The whole 137 patients were all received rituximab in combination with chemotherapy as first-line treatment. The detailed baseline patient characteristics and survival information were shown in supplementary Table S1. Kaplan-Meier analysis was performed and PLA2G2D, CCL19, and YAP1 were found to be significantly associated with OS while the expression of other five genes (MMP9, PTGDS, NFIB, RGL 1, and TIMP3) showed no significant relationship with OS (Fig. 3A-H and supplementary Figure S7). To determine independent prognostic genes, multivariate COX regression analysis was performed among the eight hub genes. Higher expression of CCL 19 and RGL 1 were significantly associated with longer OS (HR $=0.47,95 \% \mathrm{Cl}[0.25-0.86], \mathrm{p}=0.014$ for $C C L 19 ; \mathrm{HR}=0.34,95 \% \mathrm{Cl}[0.13-0.90], \mathrm{p}=0.03$ for $R G L 7$ ) (Fig. 3I).

As CCL 19 showed prognostic value in both Kaplan-Meier analysis and multivariate COX analysis in the GEO dataset, the expression of CCL 19 was further explored. Oncomine dataset analysis indicated that mRNA of CCL 19 was $\geq 84.549$ and $\geq 1.438$ fold elevated in FL samples compared to normal tissue in Compagno lymphoma dataset and Brune lymphoma dataset, respectively (Fig. 4A to B). However, in Alizadeh lymphoma dataset and Rosenwald multi-cancer dataset, the expression level of CCL 19 was showed no statistical significance between FL samples and normal tissue (Fig. 4C to D).

\section{Serum CCL19 concentration measurement}

The prognostic value of CCL 19 was further validated in CHCAMS cohort. A total of 32 pre-treatment serum samples of treatment naïve FL were included in the ELISA test, with 16 (50\%) patients were male. The median age was 47 years old. All the patients received first-line immunochemotherapy, including R$\mathrm{CHOP}$ or R-CHOP-like regimens, with 10 of $32(31.2 \%)$ receiving rituximab maintenance. Median follow-up time was 58.0 (range: 12.0-98.0) months. The detailed baseline patient characteristics and survival information were shown in supplementary Table S1. There were no difference of serum CCL19 concentration between difference age groups (mean concentration: $1.439 \mathrm{ng} / \mathrm{ml}$ in age over 60 years vs. $1.823 \mathrm{ng} / \mathrm{ml}$ in age less than 60 years, $\mathrm{p}=0.468$ ), Follicular Lymphoma International Prognostic Index risk groups (mean concentration: $2.122 \mathrm{ng} / \mathrm{ml}$ in low-risk group vs. $1.578 \mathrm{ng} / \mathrm{ml}$ in intermediated-group vs. $1.070 \mathrm{ng} / \mathrm{ml}$ in high-risk group, $p=0.348$ ) and rituximab maintenance groups (mean concentration: $1.165 \mathrm{ng} / \mathrm{ml}$ in maintenance group vs. $1.578 \mathrm{ng} / \mathrm{ml}$ in no maintenance group, $\mathrm{p}=0.278$ ) (Fig. $5 \mathrm{~A}$ to $\mathrm{C}$ ). The best-cutoff value for serum CCL19 was $0.94 \mathrm{ng} / \mathrm{ml}$ (supplementary Figure S8) and patients were grouped into high CCL19 concentration and low CCL19 concentration group according to the cutoff value. Kaplan-Meier curves showed that serum CCL19 concentration was associated with PFS and OS, with higher CCL19 concentration group having longer PFS $(p=0.014)$ and OS $(p=0.039)$ (Fig. 5D to $F)$. 


\section{CIBERSORT analysis}

To further elucidate which subset of TIICs might serve as regulatory role in different CCL 19 expression groups, CIBERSORT analysis was applied in dataset GSE119214 to calculate the relationship of CCL 19 expression and microenvironment cell subsets. Figure 6A illustrated the proportion of different subsets of TIICs between CCL 19 low expression and high expression groups. The correlations were further quantified and CCL 19 mainly had a positive correlation with monocytes $(R=0.363, \mathrm{p}<0.001)$ and macrophages $\mathrm{M} 1(R=0.325, \mathrm{p}<0.001)$, and it had a negative correlation with naïve $\mathrm{B}$ cells $(R=-0.304, \mathrm{p}$ $<0.001)$ and plasma cells $(R=-0.229, \mathrm{p}=0.007$, Fig. 6B).

\section{Discussion}

$\mathrm{FL}$ is a kind of indolent lymphoma with heterogeneity. Previous studies focused on identifying related genes using differential expression analysis. In this study, we applied WGCNA analysis utilizing RNAseq data of $F L$ and found three related co-expression gene modules, explored the gene network relationship, and identified eight key genes of the network. The prognostic value of eight key genes were validated in two independent FL patient cohorts and we identified that CCL 19 was significantly associated with PFS and OS in FL patients receiving first-line immunochemotherapy, which indicated that CCL 19 could be a potential prognosis biomarker for FL. To our best knowledge, this study is the first to report the potential prognostic value of CCL 19 in FL.

In previous studies, different genes were reported to be related to the development of FL. About over $85 \%$ FL patients were characterized by $t(14 ; 18)$, which resulted in the overexpression of BCL2 protein, a family of protein that inhibits cell apoptosis[22=. Mutations of chromatin modifying genes, including $E Z H 2$, $K M T 2 D$ and $C R E B B P$, were reported to play a complex role in $\mathrm{FL}[23=$. Mutations in linker histone genes (HIST1H1 B, C, D, and E; OCT2 [POU2F2]; IRF8; and ARID1A) were also reported to be associated with the pathogenesis of FL [24=. Besides, gene mutations including STAT6 and RRAGC were related to FL pathogenesis, which involving multiple pathways including mTOR and JAK/STAT pathways[25-27=. In recent studies, clinicogenetic risk models integrating genes for predicting prognosis of FL has been established[7, 8 = and has showed the value as the prediction model in FL management. For germinal center-derived lymphoma, including FL, moving out of germinal center and local endothelial cells is essential for dissemination to other lymph nodes or tissues[28=. Previous studies showed that interaction of lymphoma cell with chemokine played an important role in the movement of lymphoma cell through endothelial cells[29-31 = in chronic lymphocytic leukemia and classical Hodgkin lymphoma. Chemokines modulated chemotaxis in the migration and pathogenesis of FL cells was also presented in previous studies[32, 33=. In this study, among the three modules identified, the turquoise module showed significantly association with FL tumors. Functional analysis showed that genes in turquoise module was enriched in cell chemotaxis, extracellular matrix organization and extracellular structure organization. Results of our study further supported that pathway of cell interaction with chemokine may be closely related to the development of FL. 
In this study, we identified that CCL 19 was significantly associated with survival for FL patients treated with rituximab in combination with chemotherapy. CCL 19 encoded cytokine that involved in immunoregulatory and inflammatory processes. CCL19 specifically binds to chemokine receptor CCR7. CCR7 and CCL 19 played an important role in organizing thymic architecture and function, lymph-node homing of naive and regulatory $T$ cell, as well as homeostasis and inflammation-induced lymph-nodebound migration of dendritic cells, which indicated that CCR7 and CCL 19 involved in the homeostasis, immune surveillance, and tumor formation[34=. In previous studies, CCR7/CCL19 was reported to be associated with some types of cancer. CCR7 chemokine receptor binds to the ligand CCL19/CCL21 and promotes lymphogenesis and metastasis in breast cancer[35=. CCL 19 overexpression significantly inhibited gastric cancer cell proliferation and tumor growth through CCL19/CCR7/AIM2 pathway[36=. Also, CCL 19 overexpression is associated with malignant transformation in cervical cancer[37=. For lymphoma, O'Connor et al. reported that CCL19-CCR7 interactions may contributed to the increasing risk of age-related central nervous system lymphoma[38=. In T cell lymphoma, higher expression of CCR7 was associated with distant metastasis as well as tumor cell migration in vitro and the underlying mechanism might be associated with PI3K/AKT signaling pathway[39=.

In our study, CCL 19 was found to be overexpressed in different FL cell lines utilizing public database analysis. Previous studies have showed that strong in vitro chemotactic activities of CCL19 for T cells and DCs and might activate a LTa1ß2-dependent pathway of normal and pathological lymphoid tissue formation[40=. In addition, CCL19 mRNA overexpression was related to higher survival rate. Also, elevated serum CCL19 concentration was associated with longer PFS and OS. The protective prognostic value of higher CCL19 expression level had been validated at both the transcription and protein concentration in two independent FL patient cohorts. In the analysis of immune cell infiltration in tumor microenvironment, the expression of CCL19 was associated with macrophages M1 and monocyte. The protective effect of CCL19 overexpression might be explained from the mechanism of CCL19 and the formation of FL. CCL19 was produced by T-zone fibroblastic reticular cells and are essential for the formation and maintenance of the T-cell zone in lymphoid organs, as well as T cells and DCs peripheral recruitment. Therefore, elevated CCL19 expression could induce the function of T-zone reticular cells and recruit $T$ cells and DCs to tumor tissues[41=. Previous studies have reported the chemotaxis function of CCL19 for macrophages M1[42=. Also, decreased subpopulations of CD4+/CD8 $+T$ cells, macrophages and dendritic cells in patients are associated with FL transformation and are predictors of worse survival[ $43,44=$, which was consistent with the result of TIIC subtype analysis in our study.

In B cell lymphomas, including FL, DCs have showed to be correlated with better prognosis, suggesting that DCs may act against tumor cells in lymphoma [45-47=. Although in this study, the DC proportion has no significant correlation with the expression of CCL19, it is still worthwhile to exploring the function of CCL 19 and DC cells for potential clinical treatment. In fact, new generation of CAR-T cell has been engineered to expressed IL-7 and CCL19 to elevate anti-tumor efficacy[48=. Moreover, dendritic cell-based active immunotherapies [ 49 = and macrophage checkpoint inhibitor Hu5F9-G4 have showed efficacy in FL $[50=$. Result of this study indicated that $C C L 19$ could act as a biomarker to identify FL patients who may have inferior efficacy derived from first-line immunotherapy. Considering the DC and macrophages 
recruitment capability of CCL 19, it is worthwhile to explore whether these patients could benefit from dendritic cell-based active or macrophage-mediated immunotherapies for stimulating the innate immune system.

\section{Conclusion}

CCL 19 might be a potential survival biomarker for FL treated with first-line immunochemotherapy. In vitro, in vivo, and clinical studies are needed to be explored the underlying interaction and regulation mechanism of CCL19 for FL in our future studies.

\section{Declarations}

\section{Ethics approval and consent to participate}

Study of pre-treatment serum samples from patients was approved by the medical ethics committee of Cancer Hospital, CAMS \& PUMC (No. 19/088-1873).

\section{Consent for publication}

Not applicable.

\section{Competing Interests}

The authors have declared that no competing interest exists.

\section{Availability of data and materials}

Dataset GSE65135 and GSE119214 are publicly available, which can be found at https://www.ncbi.nlm.nih.gov/gds/. Data of CHCAMS cohort can be obtained from the corresponding authors under reasonable requirement.

\section{Funding}

This work is supported by Chinese Academy of Medical Sciences (CAMS) Innovation Fund for Medical Sciences (CIFMS) (Grant no. 2016-I2M-1-001).

\section{Authors' contributions}

Yuankai Shi and Xiaohong Han contributed to the study conception, design and supervision, manuscript draft, review and editing. Yu Zhou and Shasha Wang contributed to the experiment conduct, statistical data analysis, interpretation of the data, manuscript draft, review and editing. Yunxia Tao and Haizhu Chen contributed to the statistical data analysis. Ning Lou, Zhishang Zhang and Jiarui Yao contributed to the sample management and experiment conduct. All the other authors were involved in patient recruitment and data curation. Yuankai Shi and Xiaohong Han had full access to all the data in the study 
and takes responsibility for the integrity of the data and the accuracy of the data analysis. All authors contributed to the development of the article and approved the final version.

\section{Acknowledgements}

The authors thank all the patients and their families. Special thanks to Dr. Guangyu Fan and Dr. Liyuan Dai for their assistance in conducting the experiment.

\section{References}

1. Teras LR, DeSantis CE, Cerhan JR, Morton LM, Jemal A and Flowers CR. 2016 US lymphoid malignancy statistics by World Health Organization subtypes. CA Cancer J Clin. 2016; 66: 443-459. 2016/09/13. DOI: 10.3322/caac.21357.

2. Swerdlow SH, Campo E, Pileri SA, Harris NL, Stein H, Siebert R, et al. The 2016 revision of the World Health Organization classification of lymphoid neoplasms. Blood. 2016; 127: 2375-2390. 2016/03/17. DOI: 10.1182/blood-2016-01-643569.

3. Johnson PW, Rohatiner AZ, Whelan JS, Price CG, Love S, Lim J, et al. Patterns of survival in patients with recurrent follicular lymphoma: a 20-year study from a single center. J Clin Oncol. 1995; 13: 140-147. 1995/01/01. DOI: 10.1200/jco.1995.13.1.140.

4. Marcus R, Imrie K, Belch A, Cunningham D, Flores E, Catalano J, et al. CVP chemotherapy plus rituximab compared with CVP as first-line treatment for advanced follicular lymphoma. Blood. 2005; 105 : 1417-1423. 2004/10/21. DOI: 10.1182/blood-2004-08-3175.

5. Salles G, Seymour JF, Offner F, López-Guillermo A, Belada D, Xerri L, et al. Rituximab maintenance for 2 years in patients with high tumour burden follicular lymphoma responding to rituximab plus chemotherapy (PRIMA): a phase 3, randomised controlled trial. Lancet. 2011; 377: 42-51. 2010/12/24. DOI: 10.1016/s0140-6736(10)62175-7.

6. Casulo C, Byrtek M, Dawson KL, Zhou X, Farber CM, Flowers CR, et al. Early Relapse of Follicular Lymphoma After Rituximab Plus Cyclophosphamide, Doxorubicin, Vincristine, and Prednisone Defines Patients at High Risk for Death: An Analysis From the National LymphoCare Study. J Clin Oncol. 2015; 33 : 2516-2522. 2015/07/01. DOI: 10.1200/jco.2014.59.7534.

7. Pastore A, Jurinovic V, Kridel R, Hoster E, Staiger AM, Szczepanowski M, et al. Integration of gene mutations in risk prognostication for patients receiving first-line immunochemotherapy for follicular lymphoma: a retrospective analysis of a prospective clinical trial and validation in a population-based registry. Lancet Oncol. 2015; 16: 1111-1122. 2015/08/11. DOI: 10.1016/s1470-2045(15)00169-2.

8. Huet S, Tesson B, Jais JP, Feldman AL, Magnano L, Thomas E, et al. A gene-expression profiling score for prediction of outcome in patients with follicular lymphoma: a retrospective training and validation 
analysis in three international cohorts. Lancet Oncol. 2018; 19: 549-561. 2018/02/25. DOI: 10.1016/s1470-2045(18)30102-5.

9. Langfelder $P$ and Horvath S. WGCNA: an R package for weighted correlation network analysis. BMC Bioinformatics. 2008; 9: 559. 2008/12/31. DOI: 10.1186/1471-2105-9-559.

10. Langfelder P and Horvath S. Fast R Functions for Robust Correlations and Hierarchical Clustering. J Stat Softw. 2012; 46 2012/10/11.

11. Chen Y, Li ZY, Zhou GQ and Sun Y. An Immune-Related Gene Prognostic Index for Head and Neck Squamous Cell Carcinoma. Clin Cancer Res. 2021; 27: 330-341. 2020/10/25. DOI: 10.1158/10780432.Ccr-20-2166.

12. Niemira M, Collin F, Szalkowska A, Bielska A, Chwialkowska K, Reszec J, et al. Molecular Signature of Subtypes of Non-Small-Cell Lung Cancer by Large-Scale Transcriptional Profiling: Identification of Key Modules and Genes by Weighted Gene Co-Expression Network Analysis (WGCNA). Cancers (Basel). 2019; 12 2019/12/28. DOI: 10.3390/cancers12010037.

13. Liu H, Liu M, You H, Li X and Li X. Oncogenic Network and Hub Genes for Natural Killer/T-Cell Lymphoma Utilizing WGCNA. Front Oncol. 2020; 10: 223. 2020/03/21. DOI: 10.3389/fonc.2020.00223.

14. Gautier L, Cope L, Bolstad BM and Irizarry RA. affy-analysis of Affymetrix GeneChip data at the probe level. Bioinformatics. 2004; 20: 307-315. 2004/02/13. DOI: 10.1093/bioinformatics/btg405.

15. Ritchie ME, Phipson B, Wu D, Hu Y, Law CW, Shi W, et al. limma powers differential expression analyses for RNA-sequencing and microarray studies. Nucleic Acids Res. 2015; 43: e47. 2015/01/22. DOI: 10.1093/nar/gkv007.

16. Yu G, Wang LG, Han Y and He QY. clusterProfiler: an R package for comparing biological themes among gene clusters. Omics. 2012; 16: 284-287. 2012/03/30. DOI: 10.1089/omi.2011.0118.

17. Subramanian A, Tamayo P, Mootha VK, Mukherjee S, Ebert BL, Gillette MA, et al. Gene set enrichment analysis: a knowledge-based approach for interpreting genome-wide expression profiles. Proc Natl Acad Sci U S A. 2005; 102: 15545-15550. 2005/10/04. DOI: 10.1073/pnas.0506580102.

18. Walter W, Sánchez-Cabo F and Ricote M. GOplot: an R package for visually combining expression data with functional analysis. Bioinformatics. 2015; 31: 2912-2914. 2015/05/13. DOI:

10.1093/bioinformatics/btv300.

19. Shannon P, Markiel A, Ozier O, Baliga NS, Wang JT, Ramage D, et al. Cytoscape: a software environment for integrated models of biomolecular interaction networks. Genome Res. 2003; 13: 24982504. 2003/11/05. DOI: 10.1101/gr.1239303. 
20. Newman AM, Liu CL, Green MR, Gentles AJ, Feng W, Xu Y, et al. Robust enumeration of cell subsets from tissue expression profiles. Nat Methods. 2015; 12: 453-457. 2015/03/31. DOI: 10.1038/nmeth.3337.

21. Silva A, Bassim S, Sarkozy C, Mottok A, Lackraj T, Jurinovic V, et al. Convergence of risk prediction models in follicular lymphoma. Haematologica. 2019; 104: e252-e255. 2019/01/05. DOI: 10.3324/haematol.2018.209031.

22. Tsujimoto Y, Finger LR, Yunis J, Nowell PC and Croce CM. Cloning of the chromosome breakpoint of neoplastic B cells with the $t(14 ; 18)$ chromosome translocation. Science. 1984; 226: 1097-1099. 1984/11/30. DOI: 10.1126/science.6093263.

23. Green MR. Chromatin modifying gene mutations in follicular lymphoma. Blood. 2018; 131: 595-604. 2017/11/22. DOI: 10.1182/blood-2017-08-737361.

24. Li H, Kaminski MS, Li Y, Yildiz M, Ouillette P, Jones S, et al. Mutations in linker histone genes HIST1H1 $B, C, D$, and E; OCT2 (POU2F2); IRF8; and ARID1A underlying the pathogenesis of follicular lymphoma. Blood. 2014; 123: 1487-1498. 2014/01/18. DOI: 10.1182/blood-2013-05-500264.

25. Yildiz M, Li H, Bernard D, Amin NA, Ouillette P, Jones S, et al. Activating STAT6 mutations in follicular lymphoma. Blood. 2015; 125: 668-679. 2014/11/28. DOI: 10.1182/blood-2014-06-582650.

26. Okosun J, Wolfson RL, Wang J, Araf S, Wilkins L, Castellano BM, et al. Recurrent mTORC1-activating RRAGC mutations in follicular lymphoma. Nat Genet. 2016; 48: 183-188. 2015/12/23. DOI: 10.1038/ng.3473.

27. Ying ZX, Jin M, Peterson LF, Bernard D, Saiya-Cork K, Yildiz M, et al. Recurrent Mutations in the MTOR Regulator RRAGC in Follicular Lymphoma. Clin Cancer Res. 2016; 22: 5383-5393. 2016/11/03. DOI: 10.1158/1078-0432.Ccr-16-0609.

28. Linke F, Harenberg M, Nietert MM, Zaunig S, von Bonin F, Arlt A, et al. Microenvironmental interactions between endothelial and lymphoma cells: a role for the canonical WNT pathway in Hodgkin lymphoma. Leukemia. 2017; 31: 361-372. 2016/08/19. DOI: 10.1038/leu.2016.232.

29. Baekkevold ES, Yamanaka T, Palframan RT, Carlsen HS, Reinholt FP, von Andrian UH, et al. The CCR7 ligand elc (CCL19) is transcytosed in high endothelial venules and mediates T cell recruitment. J Exp Med. 2001; 193: 1105-1112. 2001/05/09. DOI: 10.1084/jem.193.9.1105.

30. Till KJ, Lin K, Zuzel M and Cawley JC. The chemokine receptor CCR7 and alpha4 integrin are important for migration of chronic lymphocytic leukemia cells into lymph nodes. Blood. 2002; 99: 29772984. 2002/04/04. DOI: 10.1182/blood.v99.8.2977.

31. Höpken UE, Foss HD, Meyer D, Hinz M, Leder K, Stein H, et al. Up-regulation of the chemokine receptor CCR7 in classical but not in lymphocyte-predominant Hodgkin disease correlates with distinct 
dissemination of neoplastic cells in lymphoid organs. Blood. 2002; 99: 1109-1116. 2002/02/07. DOI: 10.1182/blood.v99.4.1109.

32. Husson H, Carideo EG, Cardoso AA, Lugli SM, Neuberg D, Munoz O, et al. MCP-1 modulates chemotaxis by follicular lymphoma cells. Br J Haematol. 2001; 115: 554-562. 2001/12/12. DOI: 10.1046/j.1365-2141.2001.03145.x.

33. Amé-Thomas P, Maby-El Hajjami H, Monvoisin C, Jean R, Monnier D, Caulet-Maugendre S, et al. Human mesenchymal stem cells isolated from bone marrow and lymphoid organs support tumor B-cell growth: role of stromal cells in follicular lymphoma pathogenesis. Blood. 2007; 109: 693-702. 2006/09/21. DOI: 10.1182/blood-2006-05-020800.

34. Förster R, Davalos-Misslitz AC and Rot A. CCR7 and its ligands: balancing immunity and tolerance. Nat Rev Immunol. 2008; 8: 362-371. 2008/04/02. DOI: 10.1038/nri2297.

35. Rizeq B and Malki MI. The Role of CCL21/CCR7 Chemokine Axis in Breast Cancer Progression. Cancers (Basel). 2020; 12 2020/04/29. DOI: 10.3390/cancers12041036.

36. Zhou R, Sun J, He C, Huang C and Yu H. CCL19 suppresses gastric cancer cell proliferation, migration, and invasion through the CCL19/CCR7/AIM2 pathway. Hum Cell. 2020; 33: 1120-1132. 2020/06/22. DOI: 10.1007/s13577-020-00375-1.

37. Zhang $X$, Wang $Y$, Cao $Y$, Zhang $X$ and Zhao H. Increased CCL19 expression is associated with progression in cervical cancer. Oncotarget. 2017; 8: 73817-73825. 2017/11/02. DOI:

10.18632/oncotarget.17982.

38. O'Connor T, Zhou X, Kosla J, Adili A, Garcia Beccaria M, Kotsiliti E, et al. Age-Related Gliosis Promotes Central Nervous System Lymphoma through CCL19-Mediated Tumor Cell Retention. Cancer Cell. 2019; 36: 250-267.e259. 2019/09/19. DOI: 10.1016/j.ccell.2019.08.001.

39. Yang J, Wang S, Zhao G and Sun B. Effect of chemokine receptors CCR7 on disseminated behavior of human T cell lymphoma: clinical and experimental study. J Exp Clin Cancer Res. 2011; 30: 51. 2011/05/10. DOI: 10.1186/1756-9966-30-51.

40. Luther SA, Bidgol A, Hargreaves DC, Schmidt A, Xu Y, Paniyadi J, et al. Differing activities of homeostatic chemokines CCL19, CCL21, and CXCL12 in lymphocyte and dendritic cell recruitment and lymphoid neogenesis. J Immunol. 2002; 169: 424-433. 2002/06/22. DOI: 10.4049/jimmunol.169.1.424.

41. Link A, Vogt TK, Favre S, Britschgi MR, Acha-Orbea H, Hinz B, et al. Fibroblastic reticular cells in lymph nodes regulate the homeostasis of naive T cells. Nat Immunol. 2007; 8: 1255-1265. 2007/09/26. DOI: 10.1038/ni1513.

42. Xuan W, Qu Q, Zheng B, Xiong $S$ and Fan $\mathrm{GH}$. The chemotaxis of $M 1$ and $M 2$ macrophages is regulated by different chemokines. J Leukoc Biol. 2015; 97: 61-69. 2014/11/02. DOI: 10.1189/jlb.1A0314- 
170R.

43. Wahlin BE, Aggarwal M, Montes-Moreno S, Gonzalez LF, Roncador G, Sanchez-Verde L, et al. A unifying microenvironment model in follicular lymphoma: outcome is predicted by programmed death-1positive, regulatory, cytotoxic, and helper T cells and macrophages. Clin Cancer Res. 2010; 16: 637-650. 2010/01/14. DOI: 10.1158/1078-0432.Ccr-09-2487.

44. Smeltzer JP, Jones JM, Ziesmer SC, Grote DM, Xiu B, Ristow KM, et al. Pattern of CD14+ follicular dendritic cells and PD1+ T cells independently predicts time to transformation in follicular lymphoma. Clin Cancer Res. 2014; 20: 2862-2872. 2014/04/15. DOI: 10.1158/1078-0432.Ccr-13-2367.

45. Galati D, Corazzelli G, De Filippi R and Pinto A. Dendritic cells in hematological malignancies. Crit Rev Oncol Hematol. 2016; 108: 86-96. 2016/12/10. DOI: 10.1016/j.critrevonc.2016.10.006.

46. Chang KC, Huang GC, Jones D and Lin YH. Distribution patterns of dendritic cells and T cells in diffuse large B-cell lymphomas correlate with prognoses. Clin Cancer Res. 2007; 13: 6666-6672. 2007/11/17. DOI: 10.1158/1078-0432.Ccr-07-0504.

47. Tudor CS, Bruns H, Daniel C, Distel LV, Hartmann A, Gerbitz A, et al. Macrophages and dendritic cells as actors in the immune reaction of classical Hodgkin lymphoma. PLoS One. 2014; 9: e114345. 2014/12/04. DOI: 10.1371/journal.pone.0114345.

48. Adachi K, Kano Y, Nagai T, Okuyama N, Sakoda Y and Tamada K. IL-7 and CCL19 expression in CAR-T cells improves immune cell infiltration and CAR-T cell survival in the tumor. Nat Biotechnol. 2018; 36: 346351. 2018/03/06. DOI: 10.1038/nbt.4086.

49. Cox MC, Lapenta $C$ and Santini SM. Advances and perspectives of dendritic cell-based active immunotherapies in follicular lymphoma. Cancer Immunol Immunother. 2020; 69: 913-925. 2020/04/24. DOI: 10.1007/s00262-020-02577-w.

50. Advani R, Flinn I, Popplewell L, Forero A, Bartlett NL, Ghosh N, et al. CD47 Blockade by Hu5F9-G4 and Rituximab in Non-Hodgkin's Lymphoma. N Engl J Med. 2018; 379: 1711-1721. 2018/11/01. DOI: 10.1056/NEJMoa1807315.

\section{Figures}



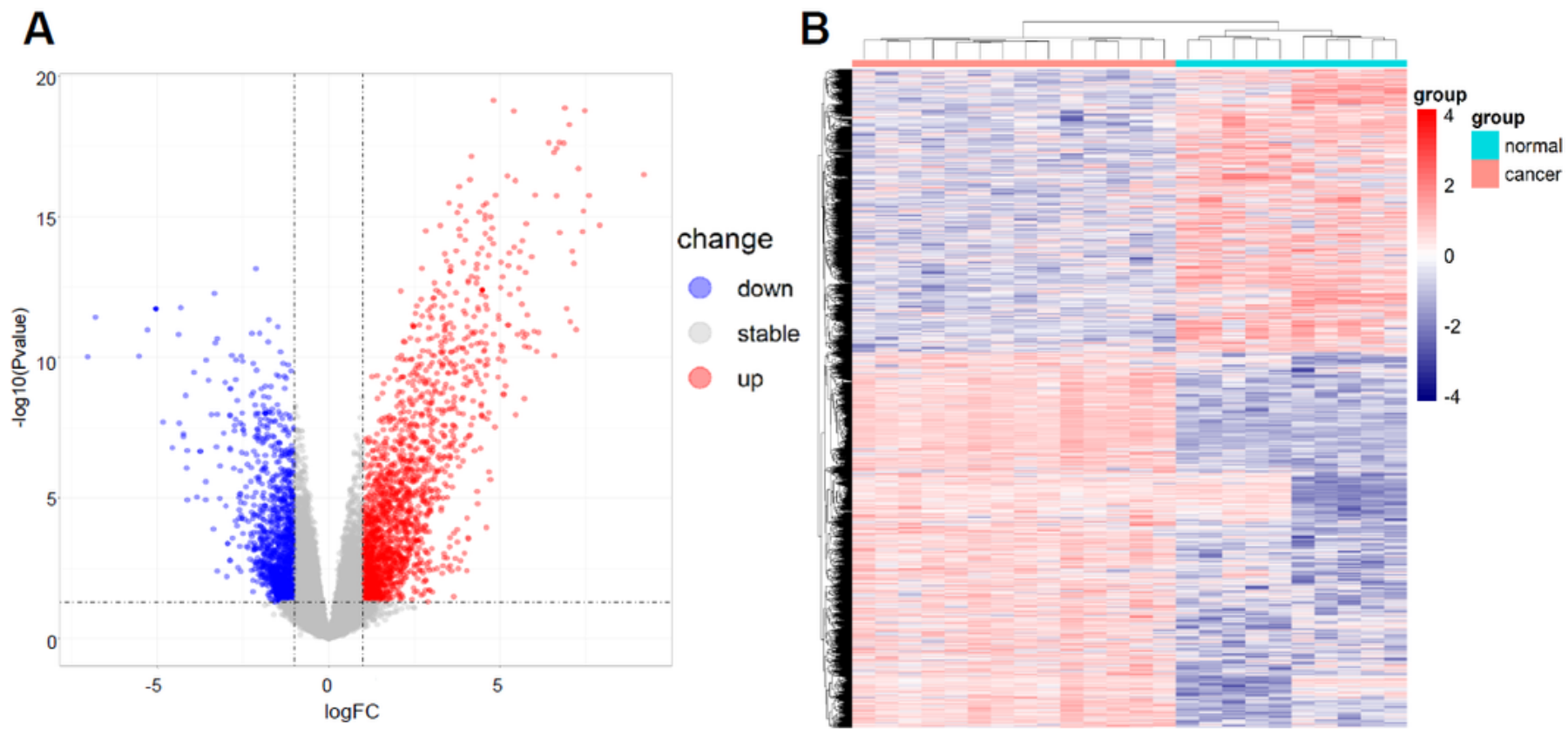

C

D
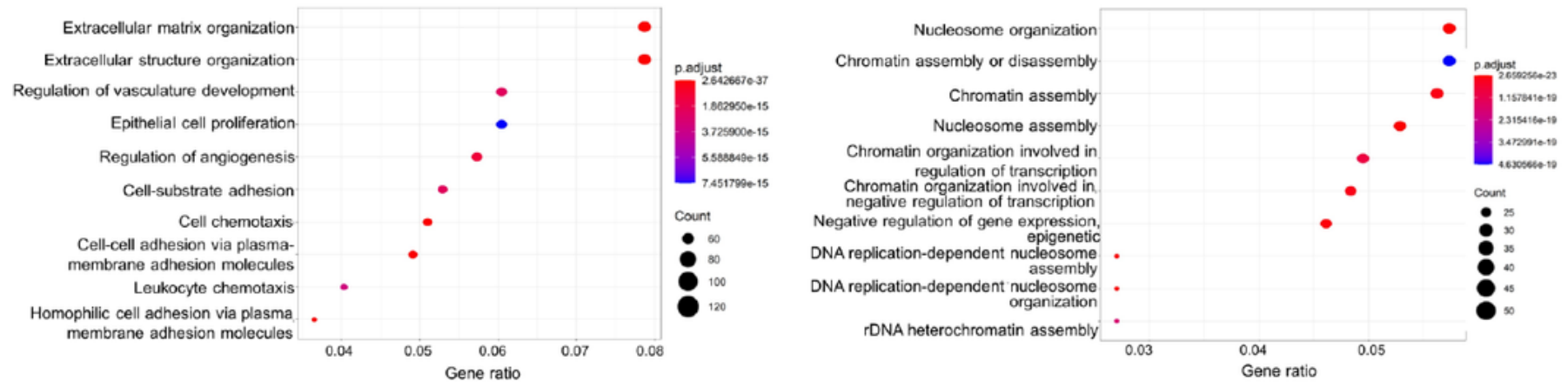

$\mathbf{E}$

$\mathbf{F}$
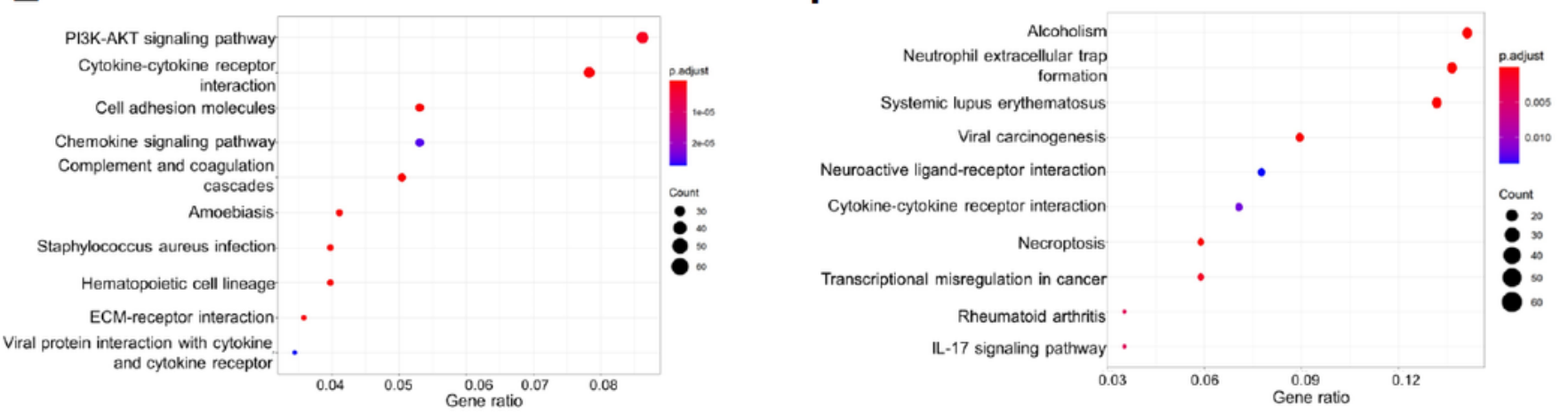

\section{Figure 1}

Differential expression profile and enrichment analysis result of follicular lymphoma. (A) Volcano plot of all differentially expressed genes (DEGs); (B) Heatmap of all DEGs between 14 tumor samples (red) and 10 normal samples (blue); (C) Top 10 Gene Ontology (GO) terms of upregulated DEGs; (D) Top 10 GO terms of downregulated DEGs; (E) Top 10 Kyoto Encyclopedia of Genes and Genomes (KEGG) pathways of upregulated DEGs; (F) Top 10 KEGG pathways of downregulated DEGs. 

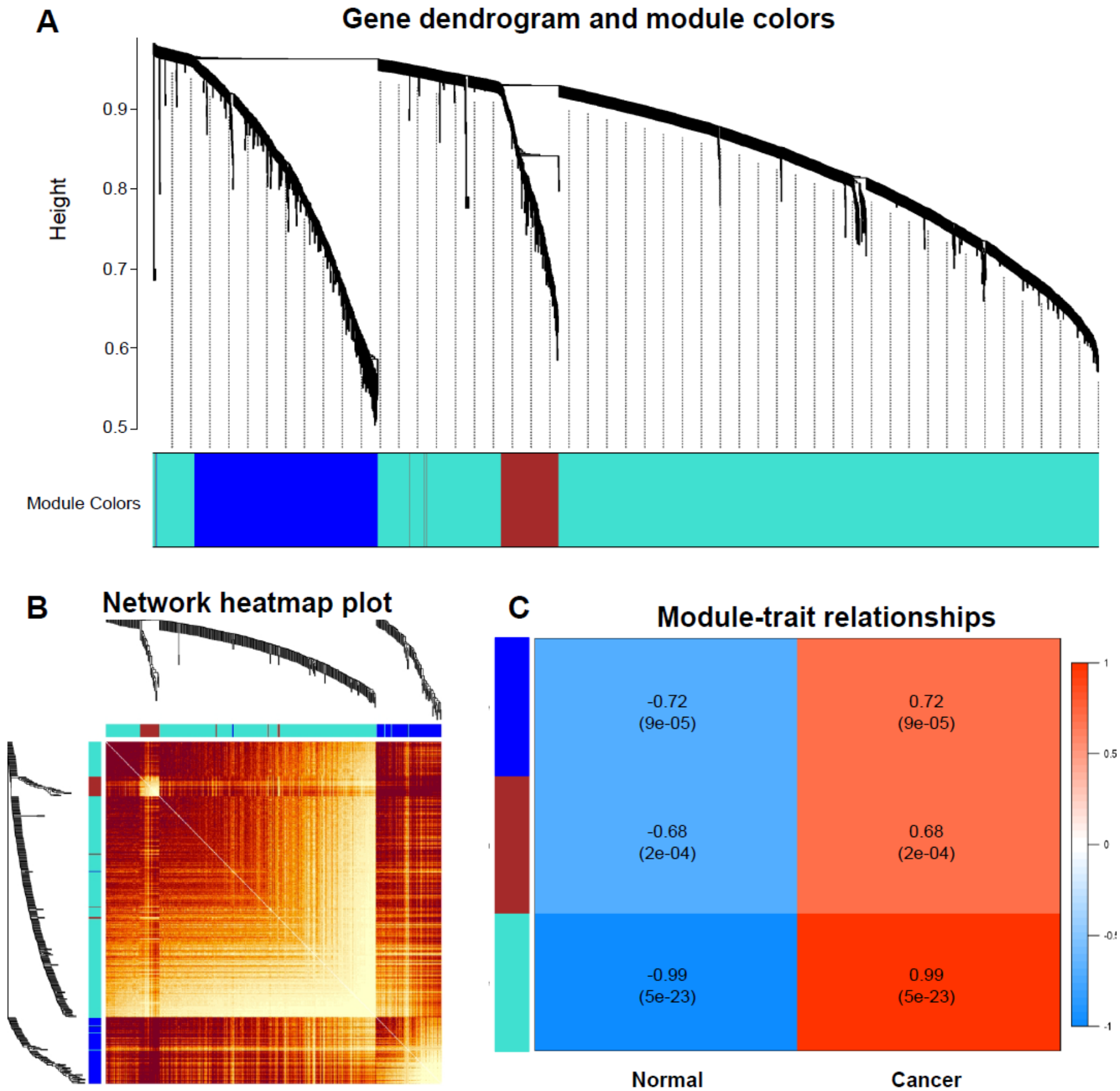

Figure 2

Weighted gene co-expression network analysis (WGCNA) for all DEGs. (A) Hierarchical cluster tree of coexpression modules identified by WGCNA. (B) Heatmap of topological overlap matrix (TOM); (C) Relationship of modules and cancer. 

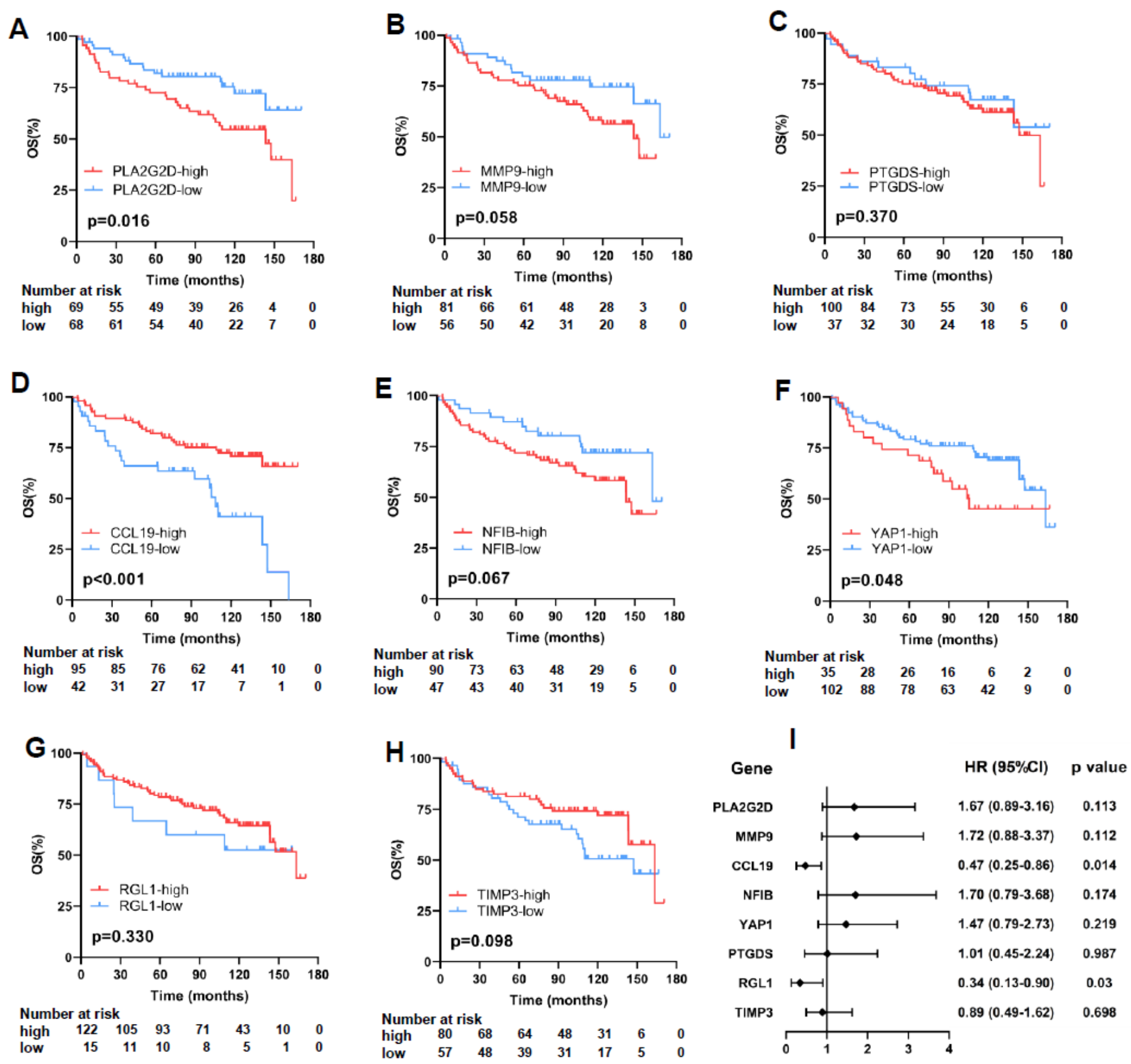

Figure 3

Kaplan-Meier survival analysis and multivariate analysis of hub genes. (A) PLA2G2D; (B) MMP9; (C)PTGDS; (D)CCL19; (E)NFIB; (F)YAP1; (G)RGL1; (H)TIMP3; (I) forest plot of multivariate Cox analysis result. 
A CCL19 Expression in Compagno Lymphoma

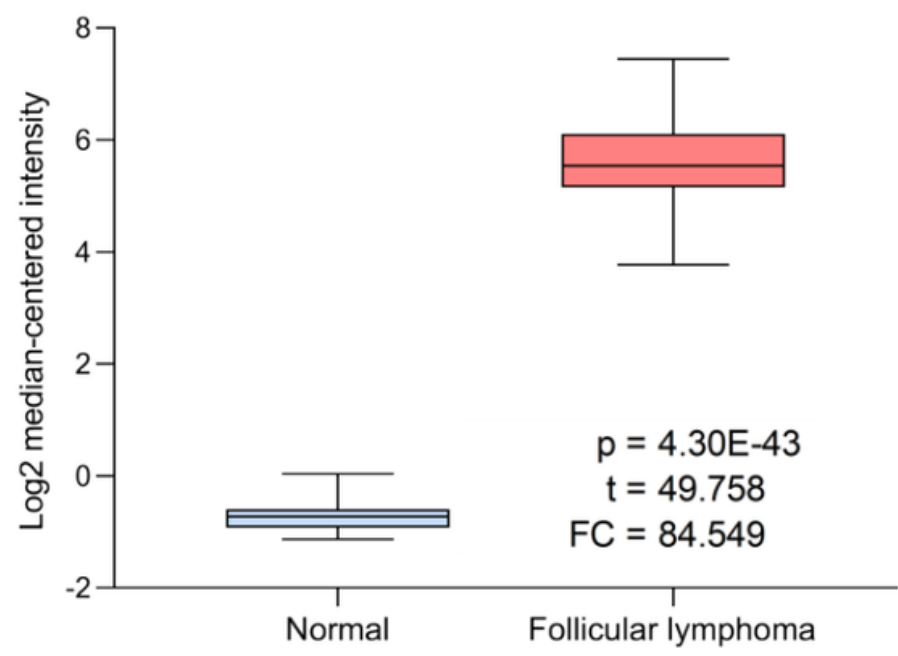

C

CCL19 Expression in Alizadeh Lymphoma

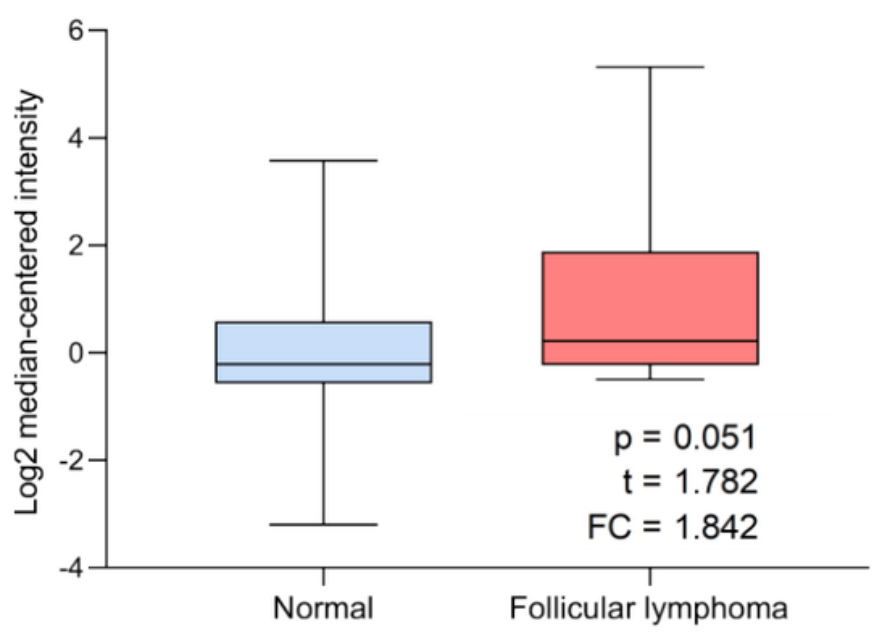

B CCL19 Expression in Brune Lymphoma

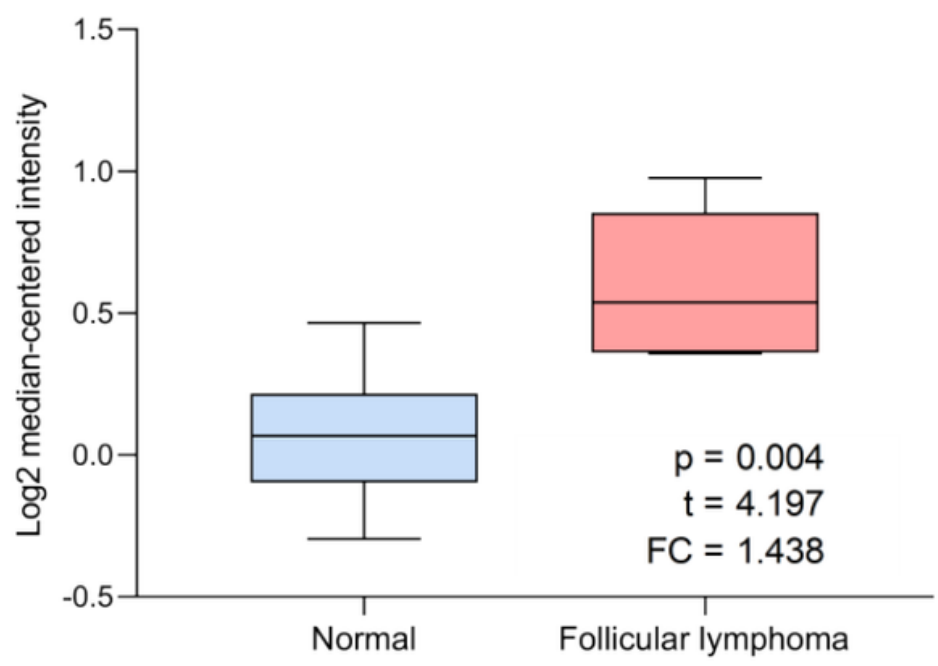

D CCL19 Expression in Rosenwald Multi-cancer

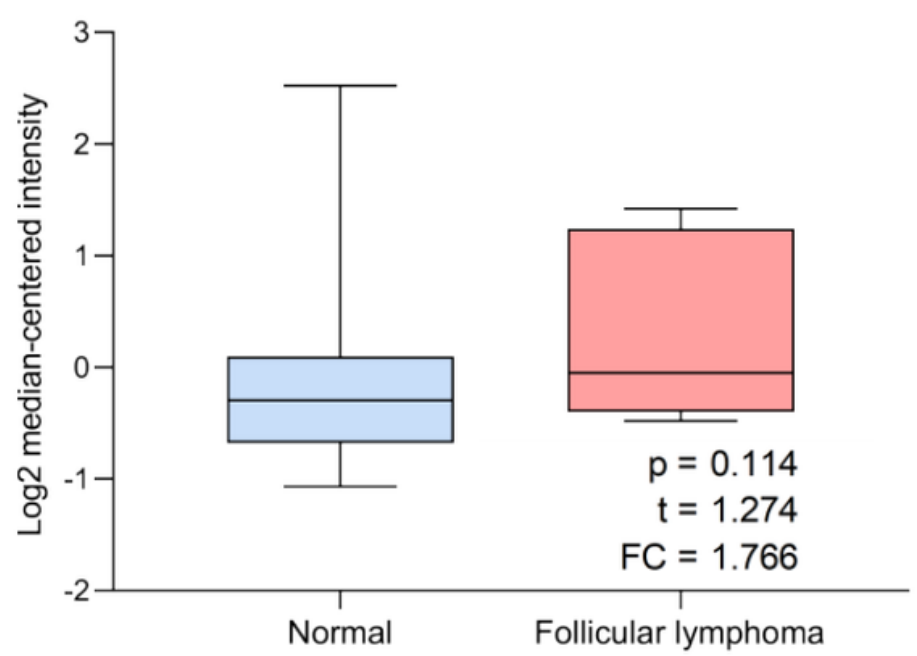

Figure 4

Box plots of CCL19 mRNA expression in Oncomine database comparing follicular lymphoma and normal tissue (A-D). 
A

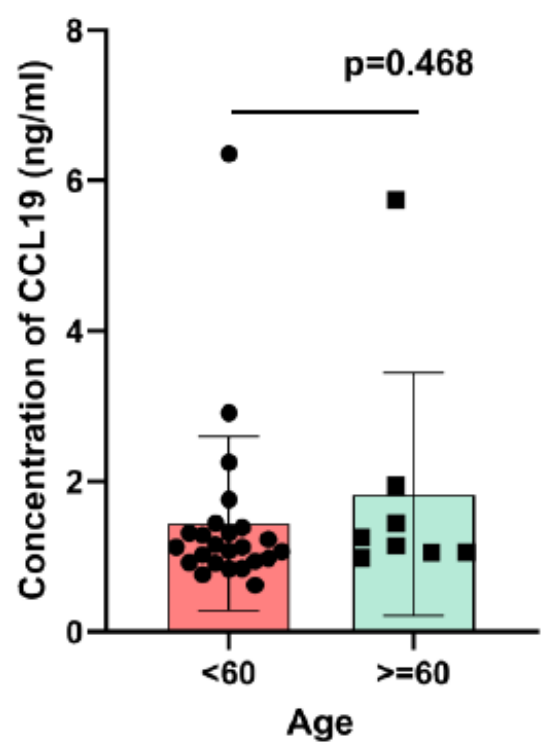

D

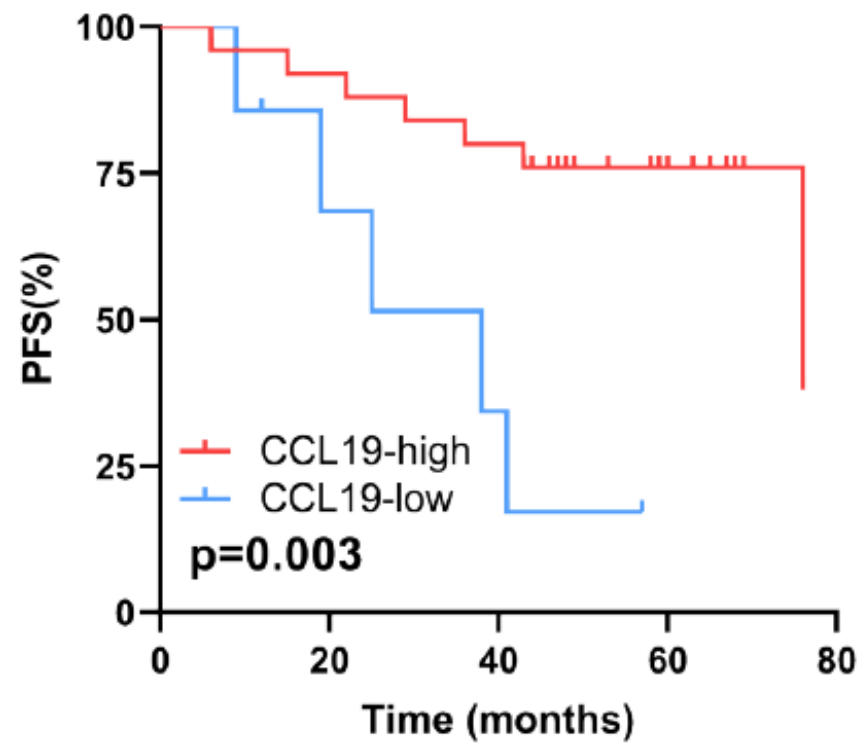

Number at risk

$\begin{array}{lccccc}\text { high } & 25 & 23 & 20 & 8 & 0 \\ \text { low } & 7 & 4 & 2 & 0 & 0\end{array}$

B
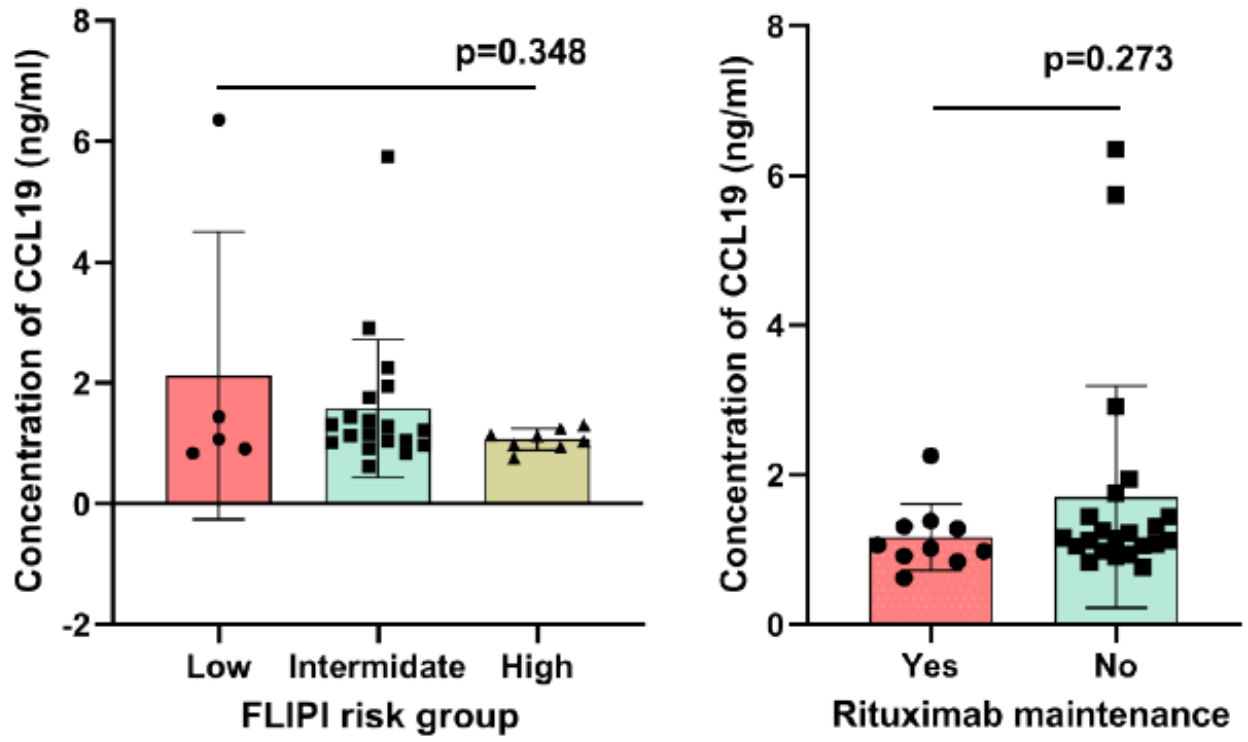

Rituximab maintenance

E

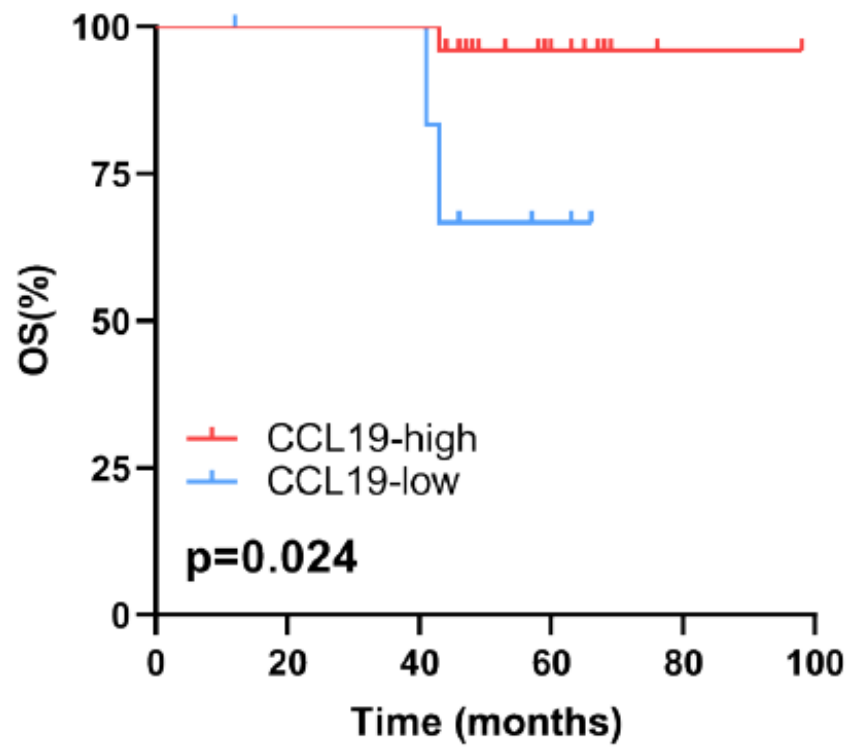

Number at risk

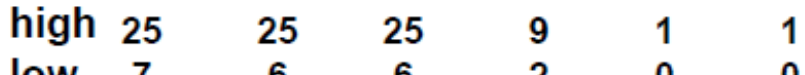

low $\begin{array}{llllll}7 & 6 & 6 & 2 & 0 & 0\end{array}$

Figure 5

The correlation of CCL19 concentration with clinical characteristics and survival outcomes.

Concentration of serum CCL19 among difference age groups (A), different FLIPI groups(B) and different rituximab maintenance groups (C). Kaplan-Meier curves of CCL19 concentration with (D) progression-free survival and (E) overall survival. Abbreviations: Follicular Lymphoma International Prognostic Index risk, FLIPI; PFS, progression-free survival; OS, overall survival. 

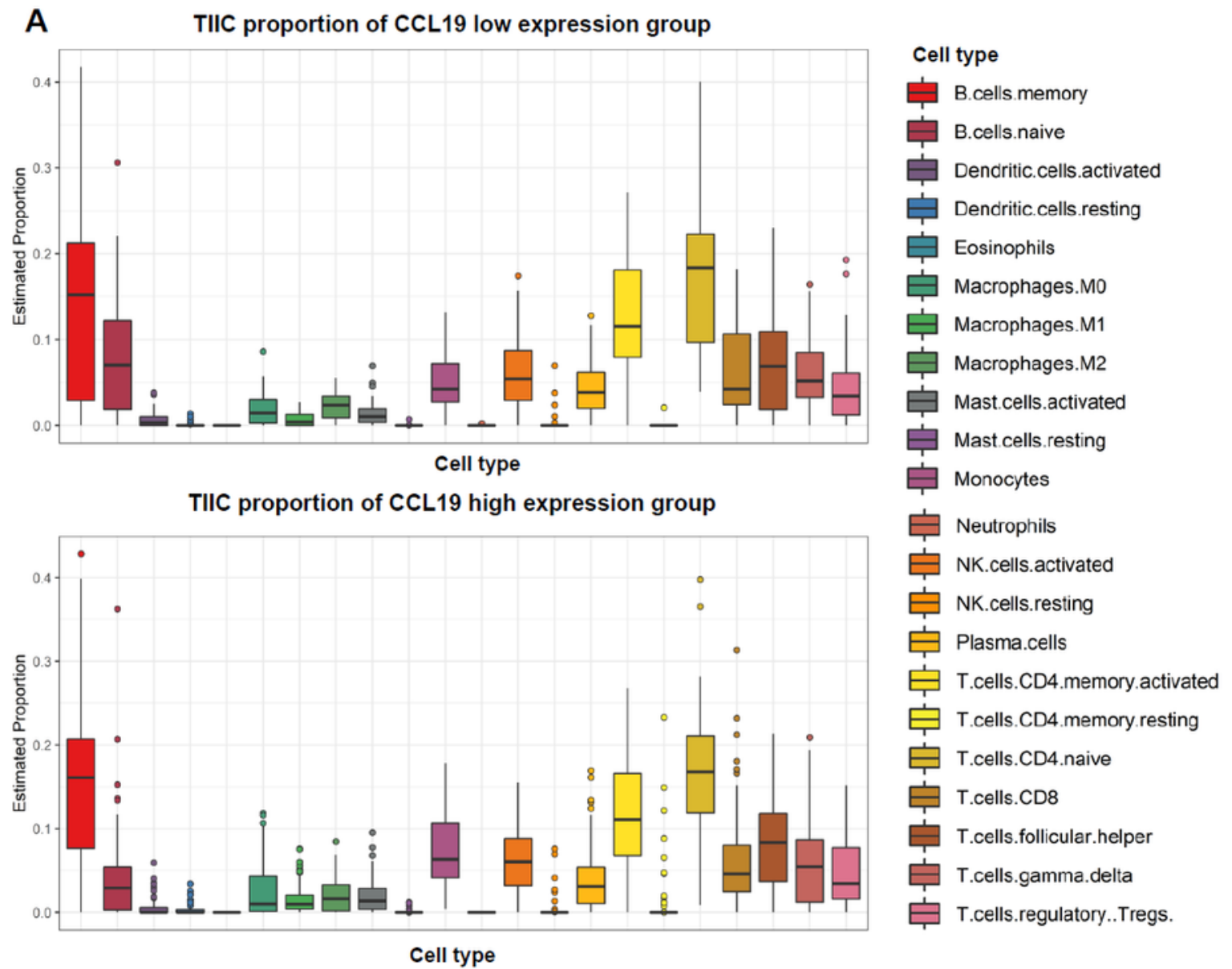

B

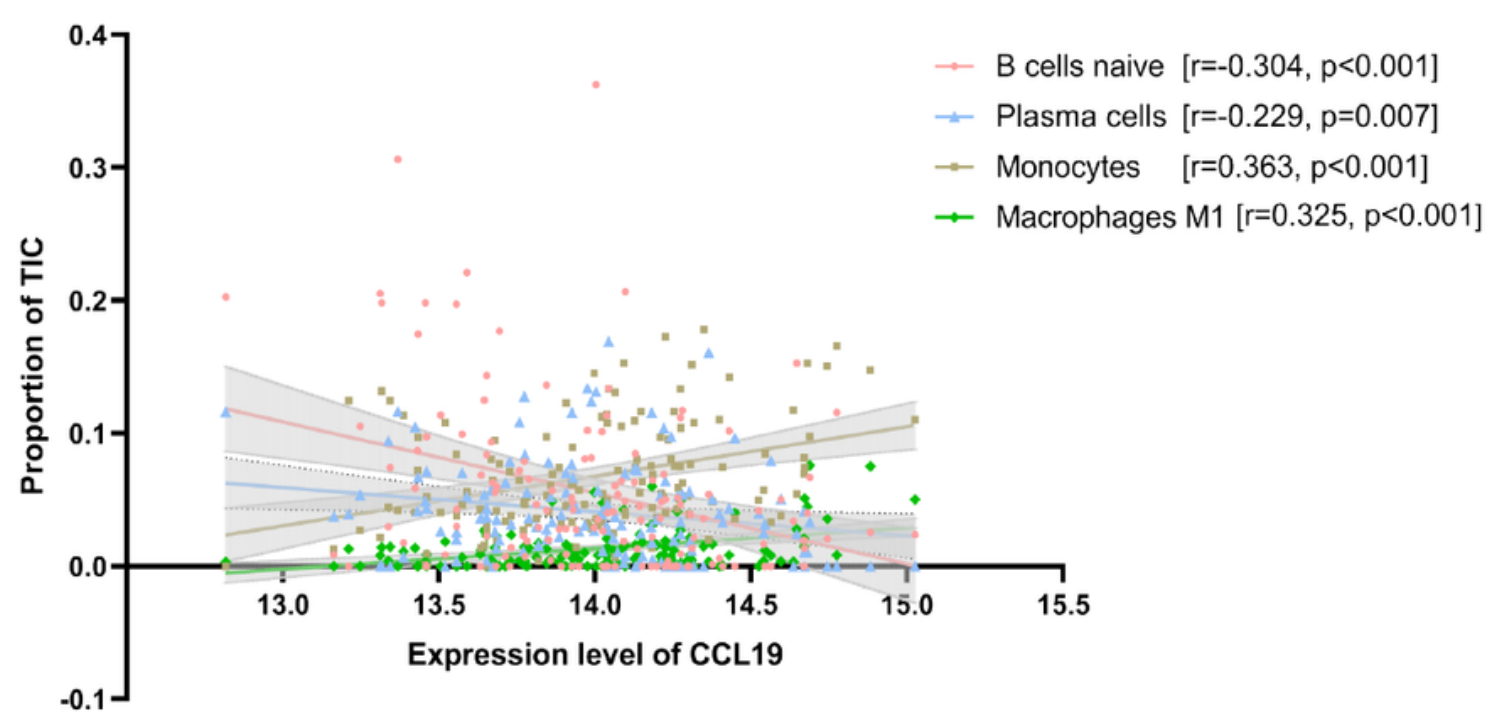

Figure 6

Results of tumor-infiltrating immune cell analysis. (A) The proportion of tumor-infiltrating immune cell (TIIC) in CCL19 low-expression (above) and high-expression (below) groups. (B) Correlation of TIIC subtypes and CCL19 expression. Abbreviations: TIIC, tumor-infiltrating immune cell.

\section{Supplementary Files}


This is a list of supplementary files associated with this preprint. Click to download.

- Supplementary.docx 